\title{
Fully 4D reconstruction of dynamic SPECT images based on the estimation of spatiotemporal basis coefficients directly from projection measurements
}

\author{
Mónica Abella, Student Member, IEEE, Bryan W. Reutter, Senior Member, IEEE, Juan Jose Vaquero, Senior \\ Member, IEEE, Manuel Desco, Grant T. Gullberg, Fellow, IEEE
}

\begin{abstract}
Quantitative kinetic analysis of dynamic cardiac SPECT data provides unique information that can enable improved discrimination between healthy and diseased tissue, compared to static imaging.

Conventional kinetic parameter estimation is based on the reconstruction of a dynamic sequence of 3D images from complete projections and the fitting of the compartmental kinetic models to time-activity curves generated by overlaying regions of interest on the reconstructed sequence. The problem with this approach is that, due to the gantry motion involved and the changes in the distribution of radiopharmaceutical during the acquisition, the projection data at different angles come from different tracer distributions resulting in inconsistent projections. Images reconstructed from these inconsistent projections can contain artifacts that lead to bias in the estimated kinetic parameters.

In this work we present a fully $4 \mathrm{D}$ reconstruction algorithm for dynamic SPECT in a parallel ray geometry. The method is based on modeling the spatial and temporal distribution of the radiotracer inside the field of view with spatial tri-linear $B$-spline and 4 piecewise quadratic temporal $B$-spline basis functions The algorithm was tested on a dynamic ${ }^{99 \mathrm{~m}}$ Tc-teboroxime cardiac SPECT/CT canine study.
\end{abstract}

\section{INTRODUCTION}

$\mathrm{K}_{\text {inetic modeling of biological processes, which has been a }}$ very active research area for many years in PET, is also generating interest in single photon emission computed tomography (SPECT). Quantitative kinetic analysis of dynamic cardiac SPECT data provides unique information that can enable improved discrimination between healthy and diseased tissue, compared to static imaging. In particular, compartmental model analysis can provide quantitative measures of myocardial perfusion, viability, and coronary flow reserve $[1,2]$.

Manuscript received November 17,2008 . This work is partially funded by the CD-TEAM Project, CENIT Program, Spanish Ministerio de Industria, with grants from the Ministerio de Educación y Ciencia, projects TEC200764731 and TEC2008-06715-C02-01, and grants R01EB00121 and R01HL71253 from the National Institutes of Health (NIH) and the U.S. Department of Energy under Contract No. DE-AC03-76SF00098.

M. Abella, J.J. Vaquero and M. Desco are with the Unidad de Medicina y Cirugía Experimental, Hospital General Universitario Gregorio Marañón, Madrid, Spain (e-mail: mabella@hggm.es). B.W. Reutter and G.T. Gullberg are with the Medical Imaging Technology Department, Lawrence Berkeley National Laboratory. Berkeley, California (bwreutter@lbl.gov).
Dynamic cardiac SPECT involves the collection of data using a rotating gamma camera (or cameras) [3]. Conventional kinetic parameter estimation is carried out after a previous reconstruction of a dynamic sequence of 3D images from complete projections acquired in relatively short time intervals. Kinetic parameters are then obtained by fitting compartmental kinetic models to time-activity curves generated from regions of interest on the temporal sequence of reconstructed images by means of a fitting routine such as RFIT (region of interest fitting) [4].

This approach presents several problems. First of all, for each reconstruction just a small part of the data is used, highly reducing the signal to noise ratio. And, more importantly, since the distribution of radiopharmaceutical changes during the acquisition, projection data at different angles come from different tracer distributions. Images reconstructed from these inconsistent projections can contain artifacts that lead to biases in the estimated kinetic parameters. To overcome these problems, several groups are developing methods to estimate the kinetic parameters or the time-activity curves directly from projection data. Direct methods estimate the kinetic model parameters directly from the projection measurements [5]. Semi-direct methods estimate first time-activity curves from the projections, and then estimate kinetic model parameters using the estimated time-activity curves [6].

In the line of the semi-direct methods, Reutter at al. [7] presented recently the extension of their work to fully 3D SPECT image reconstruction by extending the support of spatial B-spline basis functions into the time dimension. In this work we present a new approach for the 4D reconstruction algorithm for dynamic SPECT in a parallel ray geometry based on B-splines including attenuation map from CT and geometry efficiency correction. In this work, we make use of 4 piecewise quadratic temporal splines and a reconstruction algorithm based on the iterative maximization of Poisson likelihood. Results for a ${ }^{99 \mathrm{~m}} \mathrm{Tc}$-teboroxime canine study are shown.

\section{MATERIAL AND METHODS}

The main goal of this work is to obtain the time activity curves directly from the projection data. These curves will be the base for a kinetic parameter fitting method for obtaining kinetic parameters to describe the uptake and wash out of the tracer in the heart. To achieve this goal, we first obtained a 4D 
reconstruction data set which is composed of a time varying 3D reconstructed emission image. The algorithm is based on modeling the distribution of the radiotracer inside the field of view with a B-spline grid both in spatial and temporal dimensions.

\section{A. Data acquisition}

The algorithm was tested on a dynamic ${ }^{99 \mathrm{~m}} \mathrm{Tc}$-teboroxime cardiac SPECT/CT canine study. Emission data were acquired using parallel-hole collimators on a dual-head GE Millennium VG Hawkeye SPECT/CT scanner. A 30 min dynamic scan was performed, subsequent to pharmacologically induced stress as part of a rest/stress protocol.

During the scan, the gantry performed 24 360-deg rotations, acquiring 72 views per head per rotation at $1 \mathrm{sec}$ per view. Projections at each view were binned into frames of $64 \times 64$ pixels, with pixel size of $8.84 \mathrm{~mm} \times 8.84 \mathrm{~mm}$. Images were reconstructed from 64 (transverse) $\times 9$ (axial) bins corresponding to projections of the heart (Figure 1).
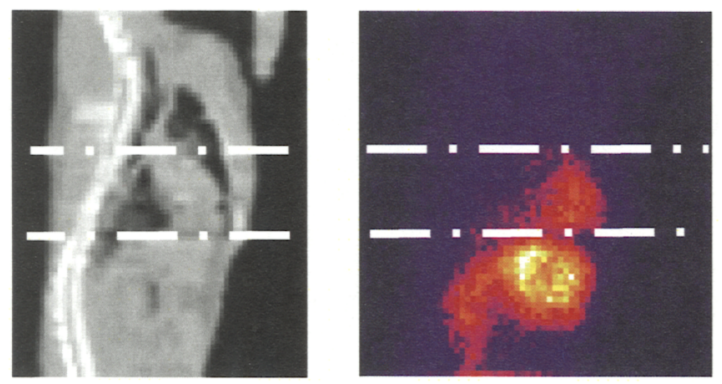

Figure 1. 64x9 bins containing the heart in a sagittal view of the reconstructed attenuation map (left), and in a late projection view (right).

An X-ray CT scan was performed with use of the integrated Hawkeye system to obtain an attenuation map.

\section{B. 4D modeling: B-splines}

The objective of this method is to obtain a 4D image that contains the information of the time evolution of the radiotracer for each point in space. To parameterize the volume, we made use of B-splines in both spatial and temporal dimensions. This allowed us to obtain a spatially and temporally continuous, smooth representation of the 4D dynamic image volume. In addition, we had a relatively small number of parameters (spline coefficients) to be estimated.

For the dynamic study we used the data from the first two rotations of the gantry, which were 144 views total. In order to reduce the dimensionality of the problem (too high if we include in the model every stage in time, which is 144 points in this case), the whole temporal variation was modeled by means of four non-uniform piecewise quadratic B-spline basis functions, shown in Figure 2.

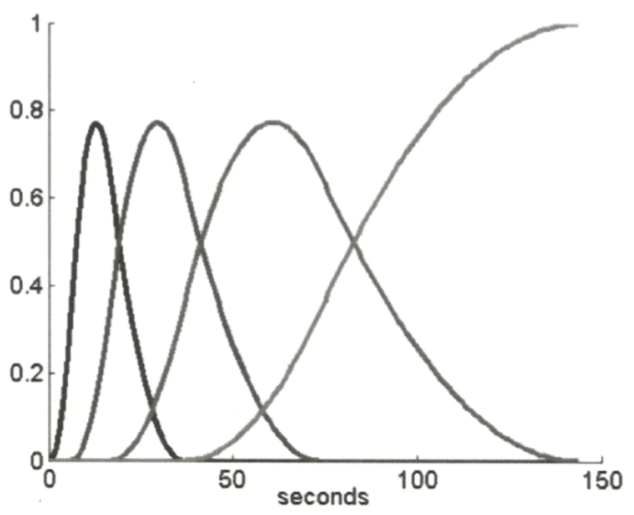

Figure 2. Four piecewise quadratic B-spline basis functions to model time variation

The result of the reconstruction process is then estimates of the coefficients for each of these four basis functions at each location of the spatial B-spline grid.

\section{Image reconstruction}

The system matrix was built in two steps. In the first step a conventional 3D system matrix was generated, including the modeling of attenuation and depth-dependent collimator response as described in [8], but not scatter. In the second step the 4D matrix was obtained as the product of the 3D matrix and the four non-uniform B-splines shown in Figure 2. The process is depicted in Figure 3.

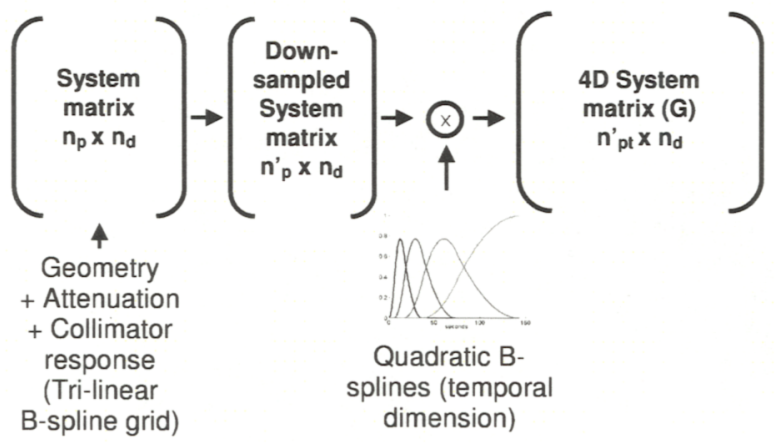

Figure 3. Process to build the 4D system matrix, where $n_{p}$ is the number of voxels in the reconstructed volume $(64 \times 64 \times 9)$, $n_{p}^{\prime}$ is the number of voxels in the downsampled volume $(32 \times 32 \times 4), n^{\prime}{ }_{p t}$ is the number of voxels in the dynamic volume $(32 \times 32 \times 4 \times 144 \times 4)$, and $n_{d}$ is the number of projection elements used $(64 \times 64 \times 72 \times 2 \times 2)$.

The reconstruction was performed using a statistical algorithm based on the maximization of the Poisson likelihood. The Ordered Subsets Expectation Maximization (OSEM) algorithm [9] was used in this application.

We obtained a late reconstructed static image ignoring the information of the few first gantry rotations, thus using the summed data in $64 \times 9$ subframes of the 72 views acquired 
during the last 22 gantry rotations. The volume was parameterized with 3D piecewise tri-linear B-splines and were organized on a $64 \times 64 \times 93 \mathrm{D}$ spatial grid that provided uniform sampling of $8.84 \mathrm{~mm} \times 8.84 \mathrm{~mm} \times 17.7 \mathrm{~mm}$ along the $x-, y-$, and $\mathrm{z}$-axes, respectively, where $\mathrm{x}$ and $\mathrm{y}$ are transverse coordinates and $\mathrm{z}$ is the axial coordinate.

The dynamic image volume was reconstructed from data in $64 \times 9$ sub-frames of the 144 dynamic views per head acquired during the first two gantry rotations. The B-splines were piecewise tri-linear in space and piecewise quadratic in time. The splines were organized on a $32 \times 32 \times 43 \mathrm{D}$ spatial grid that provided uniform sampling of $17.7 \mathrm{~mm} \times 17.7 \mathrm{~mm} \times 17.7 \mathrm{~mm}$ along the $x-, y-$, and $z$-axes, respectively, and on a 1-D temporal grid with 4 piecewise quadratic temporal B-splines.

Figure 4 shows the sampling in the projection data and the B-spline grids for each of the reconstructed volumes.

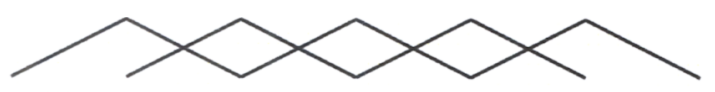

Dynamic data: $32 \times 32 \times 4$ 3D spatial grid that provided $17.6 \mathrm{~mm}$ transaxial, $17.6 \mathrm{~mm}$ axial sampling

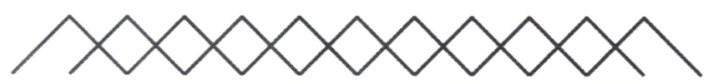

Summed data: $64 \times 64 \times 4$ 3D spatial grid that provided $8.8 \mathrm{~mm}$ transaxial and $17.6 \mathrm{~mm}$ axial sampling

$8.8 \times 8.8 \mathrm{~mm}^{2}$ pixels on the detector $(64 \times 9)$

Figure 4. Sampling in the projection data and spline grid used for each of the reconstructed volumes.

\section{RESULTS AND DISCUSSION}

Figure 5 shows the smoothed attenuation map acquired by the Hawkeye CT system and the static image reconstruction.
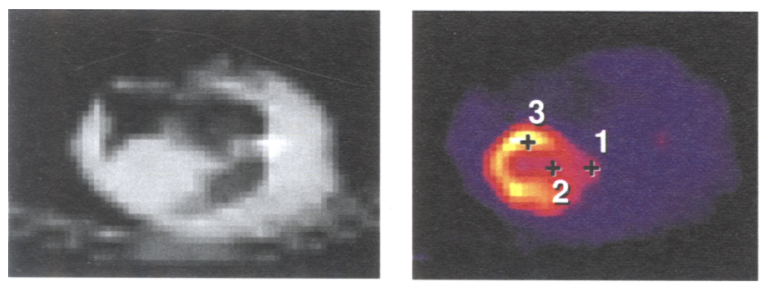

Figure 5. Left: Transaxial view of the reconstructed attenuation map from CT. Right: Transaxial view of the reconstruction of the SPECT late data (projections from the last 22 gantry rotations summed).

Figure 6 top shows time samples of a transaxial midventricular cross-section through the fully 4D dynamic reconstruction.
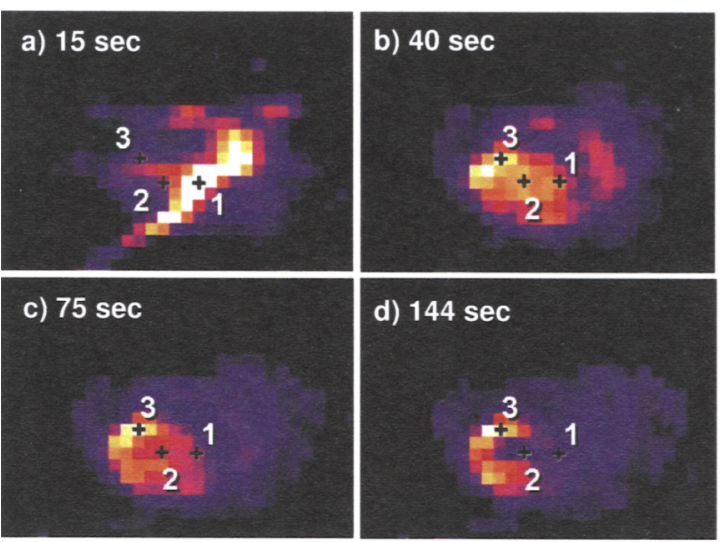

Figure 6. Central slice of the fully 4D reconstruction of dynamic early data at four different times.

The radiotracer is seen primarily in the right ventricular blood pool at $15 \mathrm{sec}$ post-injection (Fig. 6a, cross labeled "1"). At $40 \mathrm{sec}$, the radiotracer is seen primarily in the left ventricular blood pool (Fig. 6b, cross labeled "2"). Retention of the radiotracer in the left ventricular myocardium is evident at $75 \mathrm{sec}$ and $144 \mathrm{sec}$ (Figs. 6c and 6d, cross labeled "3"), as well as in the fully $3 \mathrm{D}$ reconstruction of the late summed data (Fig. 5, right). Figure 7 shows time-activity curves obtained from the $4 \mathrm{D}$ dynamic reconstructions for the three points.

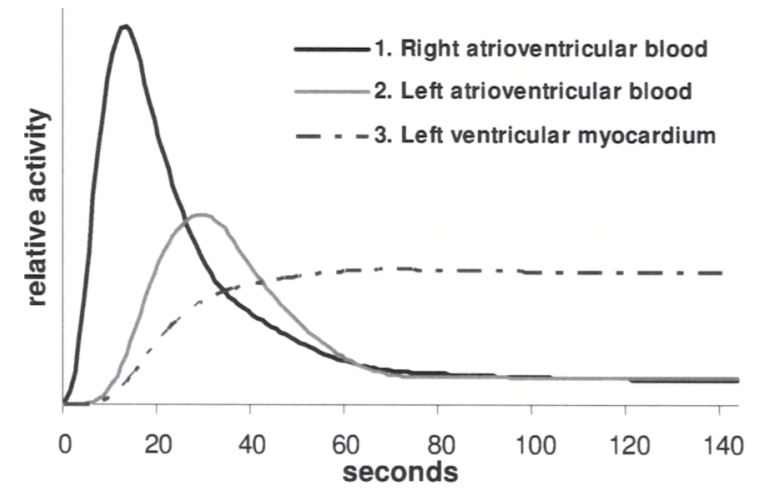

Figure 7. Time activity curves for the right atrio-ventricular blood pool, left atrio-ventricular blood pool, and left ventricular myocardium corresponding to points 1, 2, 3 in Figure 6.

We have presented a fully 4D reconstruction algorithm for dynamic SPECT in a parallel ray geometry with a gantry rotating at less than one revolution per minute. The method is based on modeling the spatial and temporal distribution of the radiotracer inside the field of view with spatial tri-linear Bspline and 4 piecewise quadratic temporal B-spline basis functions. The reconstruction is performed by means of the iterative maximization of the Poisson likelihood, with an OSEM algorithm. 
For the fully 4D dynamic ${ }^{99 \mathrm{~m}} \mathrm{Tc}$-teboroxime cardiac SPECT reconstruction, the use of non-uniform time sampling with 4D B-splines that varied quadratically in time yielded smooth time-activity curves that captured the relatively fast rise and fall of radiotracer in the right and left blood chambers, as well as uptake and retention of radiotracer in the left ventricular myocardium.

\section{REFERENCES}

1. Gullberg, GT, Huesman, RH, Ross, SG, Di Bella, EVR, Zeng, GL, Reutter, BW, Christian, PE, and Foresti, SA, 1999, "Dynamic cardiac single-photon emission computed tomography", in Nuclear Cardiology: State of the Art and Future Directions, B, BL Zaret and GA Belter, Eds., Mosby, St. Louis, pp 137-187.

2. Di Bella, EVR, Ross, SG, Kadrmas, DJ, Khare, HS, Christian, PE, McJames, S, and Gullberg, GT, 2001, "Compartmental modeling of technetium-99m-labeled teboroxime with dynamic single-photon emission computed tomography: Comparison with static thallium201 in a canine model", Invest. Radiol, 36(3), pp 178185.

3. Gullberg, GT, 2004, "Dynamic SPECT imaging: exploring a new frontier in medical imaging". in ISBI2004 IEEE International Symposium on Biomedical Imaging, Arlington, VA, pp 607-610.

4. Huesman, RH, Knittel, BL, Mazoyer, BM , Coxson, PG, Sameron, EM, Klein, GJ, Reutter, BW, and
Budinger, TF, "Notes on RFIT: A program for fitting compartment models to region-of-interest dynamic emission tomography data". 1995: Lawrence Berkeley Laboratory Report Number: LBL-37621, version 4.3.

5. Huesman, RH, Reutter, BW, Zeng, GL, and Gullberg, GT, 1998, "Kinetic parameter estimation from SPECT cone-beam projection measurements", Phys. Med. Biol., 43, pp 973-982.

6. Reutter, BW, Gullberg, GT, and Huesman, RH, 2000, "Direct least squares estimation of spatiotemporal distributions from dynamic SPECT projections using a spatial segmentation and temporal B-splines", IEEE Trans. on Med. Imag., 19(5), pp 434-450.

7. Reutter, BW, Gullberg, GT, Boutchko, R, Balakrishnan, K, Botvinick, EH, and Huesman, RH, 2007, "Fully 4-D dynamic cardiac SPECT image reconstruction using spatiotemporal B-spline voxelization". in Nuclear Science Symposium Conference Record, 6, pp 42174221.

8. Zeng, GL, Gullberg, GT, Terry, JA, and Tsui, BMW, 1991, "Three-dimensional iterative reconstruction algorithms with attenuation and geometric point response correction", IEEE Trans. on Nucl. Sci., 38(2), pp 693-702.

9. Hudson, HM and Larkin, RS, 1994, "Accelerated image reconstruction using ordered subsets of projection data", IEEE Trans. on Med. Imag., 13(4), pp 601-609. 